\title{
Graph Method for Prioritizing Capabilities of the Armed Forces
}

\author{
Rafal Kasprzyk $^{1, *}$, Zbigniew Tarapata ${ }^{1}$ \\ ${ }^{1}$ Institute of Computer and Information Systems, Faculty of Cybernetics, Military University of Technology, Warsaw, Poland
}

\begin{abstract}
The paper presents a novel method to prioritize capabilities of Polish Armed Forces, used in one of the most important stages of planning process i.e. "Required Capabilities Assessment and Prioritization". The current approach to planning and programming of the capabilities development of the Armed Forces of the Republic of Poland is executed on the basis of the so-called operational capabilities. The operational capability is defined as "potential competence, feature of an entity resulting from its characteristics, allowing to undertake actions aimed at producing the desired effects". The elements of functional operational capabilities are as follows: doctrines, organization, training, military equipment, human resources, leadership, infrastructure and interoperability. The capabilities of the Armed Forces of the Republic of Poland de facto determine executability of the military tasks. In the paper capability dependency graph model was presented and centrality measures were described in details, which are the basis for prioritization method. To manage the capabilities of the Armed Forces of the Republic of Poland, the software for prioritizing capabilities consisting of the Editor of the Capabilities Directory and Prioritization of Capabilities component, was developed and described in the paper.
\end{abstract}

\section{Introduction}

In every developed country, the armed forces constitute a fundamental defence component intended for enforcing the security policy. To efficiently accomplish such mission, it is necessary to determine appropriate directions of development of the armed forces so that they are ready to react to the most probable threats to national security. Methodology of planning and programming development of the Armed Forces of the Republic of Poland [1] is a valid document ordering the development process of the Armed Forces of the Republic of Poland. The planning of development of the Armed Forces refers to the definition of development goals, whereas the programming process consists in the preparation of various concepts for achieving such goals. Fig. 1 shows the concept of planning and programming the capabilities of the Armed Forces of the Republic of Poland.

The current approach to planning and programming of the capabilities development of the Armed Forces of the Republic of Poland is executed on the basis of the socalled operational capabilities [1], [2], [3]. The operational capability is defined as "potential competence, feature of an entity resulting from its characteristics, allowing to undertake actions aimed at producing the desired effects". The elements of functional operational capabilities are as follows: doctrines, organization, training, military equipment, human resources, leadership, infrastructure and interoperability. The capabilities of the Armed Forces of the Republic of Poland de facto determine executability of the military tasks.

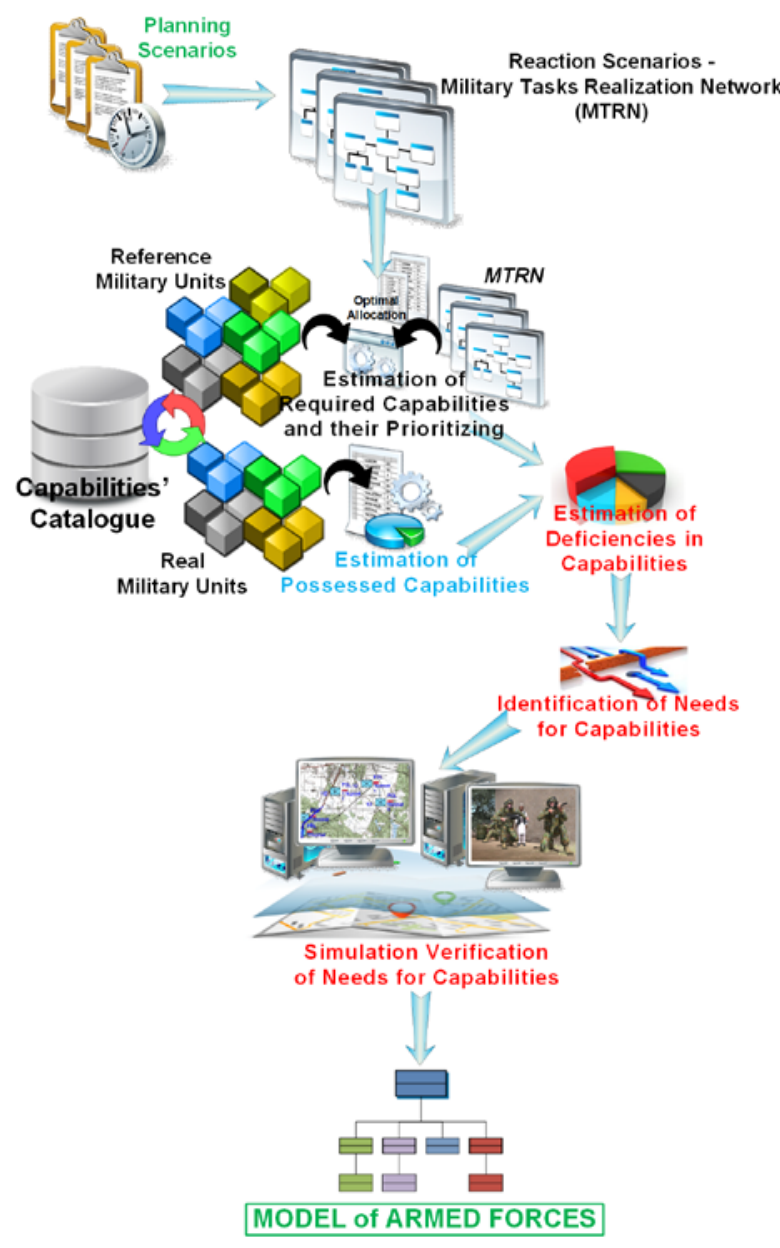

Fig. 1. Planning and programming of the capabilities development of the Armed Forces of the Republic of Poland

* Corresponding author: rafal.kasprzyk@,wat.edu.pl 
The method for prioritizing capabilities of the Armed Forces was developed for the purpose of analytical support of the decision-makers, while they perform one of the key actions in the planning process, i.e. "Assessment of the required capabilities and their prioritization". The necessity to prioritize the capabilities results from limited, especially financial, resources, within the time horizon (usually 10 years) analysed as part of the capabilities development planning process.

\section{Formal frameworks of the method for prioritizing capabilities}

The graph showing capability interdependencies between different capabilities, designed for the established Threat Reaction Scenario of the Armed Forces in case of a given risk, constitutes grounds for prioritizing capabilities. It is planned to use for that purpose the knowledge of experienced military experts, who shall be able to determine interdependencies between various capabilities. The capability dependency graph constitutes grounds for determining the so-called central capability, which facilitates quantitative approach to capabilities prioritization for a specific Threat Reaction Scenario of the Armed Forces.

\subsection{Definitions and notations}

The capability dependency graph $C D G$ is defined as an ordered two:

$$
C D G=\langle C, D\rangle
$$

where:

$C$ - a set of graph $C D G$ vertices, representing capabilities,

$D$ - a set of graph $C D G$ arcs (directed adges), representing interdependencies between capabilities, $D \subset\{\langle x, y\rangle: x, y \in C\}$. The arc $\langle x, y\rangle$ direction allows to make a distinction between the supporting capability $-x$ and supported capability $y$ for a given pair of $\langle x, y\rangle$ capabilities (see Fig. 2).

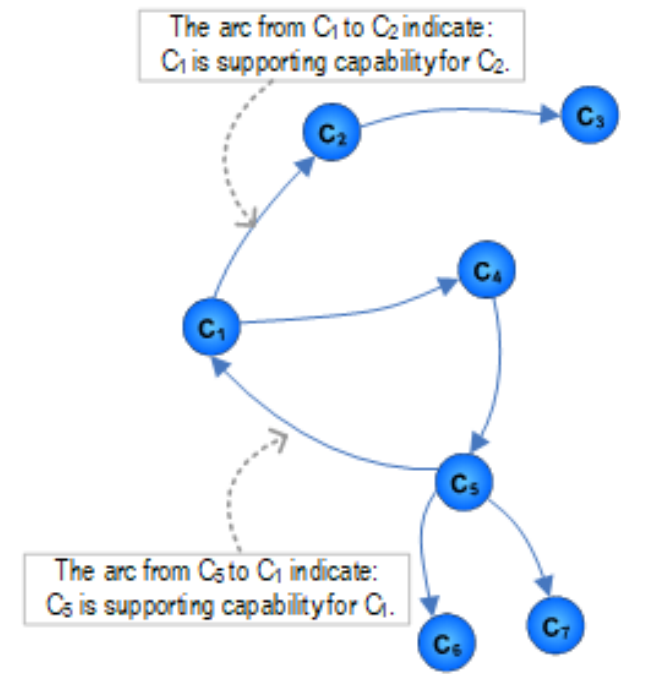

Fig. 2. Graphical representation of capability dependency graph

\subsection{Measures for assessing centrality of vertices}

The developed method for prioritizing capabilities is based on the specialized algorithms for assessing centrality of vertices in graphs, used, among other things, to search for key indyvidual in social networks (e.g. degree, radius, closeness, betweenness) and to create rankings of websites (e.g. PageRank, HITS). The centrality measures allow to answer the question which vertice is the most important in the analyzed graph from the point of view of the adopted perspective [5], [6]. Therefore, the centrality measures are often called significance measures. In the subsequent part hereof, the methods for determining the value of a specific centrality measure of vertices (capabilities) for the capability dependency graph $C D G$ are presented.

\subsubsection{Normalized in-degree centrality, $d c_{i}^{I N}$}

The significance of capabilities is proportional to the number of the supporting capabilities. The higher value of the measure, the more important the supported capability:

$$
d c_{i}^{I N}=\frac{k_{i}^{I N}}{|C|-1}
$$

where: $k_{i}^{I N}$ - the in-degree of the $i^{\text {th }}$ vertice.

\subsubsection{Normalized out-degree centrality, $d c_{i}^{\text {OUT }}$}

The significance of capabilities is proportional to the number of the supported capabilities. The higher value of the measure, the more important the supporting capability:

$$
d c_{i}^{\text {OUT }}=\frac{k_{i}^{\text {OUT }}}{|C|-1}
$$

where: $k_{i}^{\text {OUT }}$ - the out-degree of the $i^{\text {th }}$ vertice.

\subsubsection{Normalized degree centrality, $d c_{i}$}

Sometimes, the overall significance of the capabilities, both supported and supporting, may be of interest to us; in such case, the number of capabilities, through which the capability is supported and which it supports, shall be estimated:

$$
d c_{i}=\frac{k_{i}^{I N}+k_{i}^{O U T}}{2 \cdot(|C|-1)}
$$

\subsubsection{Radius centrality, $r c_{i}$}

According to this measure, the more important is the capability, the shortest is its distance (route length) to the furthest vertice (capability) on the graph. The measure is very useful to obtain the capability with the strongest support (usually indirect) for the most distant supported capability: 


$$
r c_{i}=\frac{1}{\max _{j \in C} d_{i j}}
$$

where: $d_{i j}$ - the shortest route on the $C D G$ graph from $i$ to $j$ vertices.

\subsubsection{Closeness centrality, $c c_{i}$}

According to this measure, the more important is the capability, the smaller is the total distance of its routes to all graph vertices. The capability which provides the greatest support to all other capabilities is the most important supporting capability:

$$
c c_{i}=\frac{|C|-1}{\sum_{j \in C} d_{i j}}
$$

\subsubsection{Betweenness centrality, $b c_{i}$}

Another method for assessing the capability significance is the measure, whose value is proportional to the percentage of the number of the shortest routes between each pair of vertices crossing the analyzed vertice.

$$
b c_{i}=\frac{\sum_{l \in C} \sum_{k \neq l \in C} \frac{p_{l, i, k}}{p_{l, k}}}{(|C|-2)(|C|-1)}
$$

where:

$p_{l, k}$ - the number of the shortest routes from vertice $l$ to vertice $k$,

$p_{l, i, k}$ - the number of the shortest routes from vertice $l$ to vertice $k$, crossing vertice $i$.

\subsubsection{Prestige centrality, $p c_{i}$}

Even though the $d c_{i}^{I N}$ measure used to assess the significance of the supported capability, considers only the number of the capabilities supporting a given capability, it is evident that not all supporting capabilities should have the same impact on the significance of the supported capability. If the capability has a substantial number of the supporting capabilities, which, however, are not significance, such capability should not be considered so important as the capability which is supported by at least one, yet significant supporting capability. This line of reasoning constitutes grounds for the algorithm used to set prestige centrality of the vertices (capabilities) :

$$
p c_{i}=\sum_{j \in C} a_{j i} \cdot p c_{j}
$$

where:

$a_{j i}$ - element in row $j$ and column $i$ of the state-transition matrix $A$ on the $C D G$ graph. The $a_{j i}=1 \Leftrightarrow$ element of capability $j$ is the capability supporting capability $i$ (i.e. there is an arc from vertice $j$ to vertice $i$ ), otherwise $a_{j i}=0$.
The prestige centrality vector may be established based on the eigenvector of the state-transition matrix of graph $A$. Using the matrix notation, it is possible to note:

$$
\overrightarrow{p c}=\frac{1}{\lambda} A^{T} \cdot \overrightarrow{p c}
$$

The $\overrightarrow{p c}$ vector is the eigenvector of matrix $A$ for the largest eigenvalue $\lambda$.

\subsubsection{PageRank, prc}

A subtle modification of the algorithm for setting the prestige centrality value is the PageRank algorithm, widely used to create website rankings. The concept of such algorithm consists in considering the factor related to the distribution of the impact of significance of the supporting capability on the supported capabilities. Therefore, if a given capability supports many capabilities, its impact on the significance of the supported capability is decreased:

$$
\operatorname{prc}_{i}=\sum_{j \in C} \frac{a_{j i}}{k_{j}^{\text {OUT }}} \cdot \operatorname{prc}_{j}
$$

where: $k_{j}^{\text {OUT }}$ - the out-degree of the $j^{\text {th }}$ vertice

The vector of the value of PageRank vertices may be determined based on the eigenvector of the modified $M A$ state-transition matrix on the $C D G$ graph. The element of the $M A$ matrix in the $i^{\text {th }}$ row and $j^{\text {th }}$ column shall be determined in the following manner: $m a_{i j}=\frac{a_{i j}}{k_{i}^{O U T}}$. Using the matrix notation, it is possible to note:

$$
\overrightarrow{p r c}=\frac{1}{\lambda_{M A}} M A^{T} \cdot \overrightarrow{p r c}
$$

The $\overrightarrow{p r c}$ vector is the eigenvector of matrix $M A$ for the largest eigenvalue $\lambda_{M A}$.

\subsubsection{HITS}

The HITS algorithm constitutes a subtle modification of the algorithm (with respect to PageRank) for setting prestige centrality and allows to distinguish between the values of the so-called authority centrality and hub centrality. The vertice (capability) is the more important authority (supported capability), the more it is indicated by the significant hub centrality (supporting capability). Additionally, the vertice is the more important hub centrality, the more important authority it indicates. If $a c_{i}$ and $h c_{i}$ are used to select the values of authority and hub centrality of vertice $i$, respectively, then, the following shall be obtained:

$$
\left\{\begin{array}{l}
a c_{i}=\frac{1}{\lambda_{a}} \sum_{j \in C} a_{j i} \cdot h c_{j} \\
h c_{i}=\frac{1}{\lambda_{h}} \sum_{j \in C} a_{i j} \cdot a c_{j}
\end{array}\right.
$$

Using the matrix notation, it is possible to note: 


$$
\left\{\begin{array} { l } 
{ \vec { a c } = \frac { 1 } { \lambda _ { a } } A ^ { T } \cdot \vec { h c } } \\
{ \vec { h c } = \frac { 1 } { \lambda _ { h } } A \cdot \vec { a c } }
\end{array} \Rightarrow \left\{\begin{array}{l}
\overrightarrow{a c}=\frac{1}{\lambda_{a}} A^{T} A \cdot \overrightarrow{a c} \\
\overrightarrow{h c}=\frac{1}{\lambda_{h}} A A^{T} \cdot \overrightarrow{h c}
\end{array}\right.\right.
$$

The $\overrightarrow{a c}(t)$ and $h \overrightarrow{c(t)}$ vector is the eigenvector of the $A^{T} A$ matrix for the largest $\lambda_{a}$ and $A A^{T}$ and $\lambda_{h}$ values, respectively.

\section{Software components for prioritizing capabilities}

\subsection{Editor of the Capabilities Directory}

To manage the capabilities of the Armed Forces of the Republic of Poland, an original program component i.e. the Editor of the Capabilities Directory, presenting the capabilities defined with a fully editable tree describing the taxonomy of capabilities, was developed. Feasible actions (adding new secondary capabilities, editing, distinguishing and removing capabilities) are assigned to each capability at any level of the tree. The capability may have the "distinguishing" status assigned both from the level of the capability tree, using the aforesaid actions, as well as from the level of a window for adding and editing capabilities. The "distinguishing" status was introduced to facilitate the management of the Capabilities Directory by indicating the capabilities that need special attention, for any reason. Additionally, the "temporary" status was added to suggest the temporary condition of a given capability. The status is used when the description of a capability is not fully specified or it is difficult to classify and locate the capability on the capability tree structure.

When writing the Capabilities Directory into a database, it is possible to state whether the directory is "valid" or not at a given moment, i.e. whether it is effective in the current planning cycle. In the Prioritization of Capabilities component (described in point 3.2), the Capabilities Directory is used by default and checked as "valid". It is also worth mentioning that the Editor of the Capabilities Directory makes it possible to define many Capabilities Directories, which allows to carry out the works simultaneously by independent groups of military experts while developing appropriate taxonomy of capabilities and describing them for the subsequent planning cycle.

\subsection{Prioritization of Capabilities}

For the purpose of constructing the capability dependency graph, the original software component (Fig. 3) allowing interactive construction of the graph was developed. The capability tree describing the adopted taxonomy of capabilities in a given planning cycle (area number 1) and the working area, which is the container for the constructed graph (area number 2, see also Fig. 3), constitute key elements of the editor.

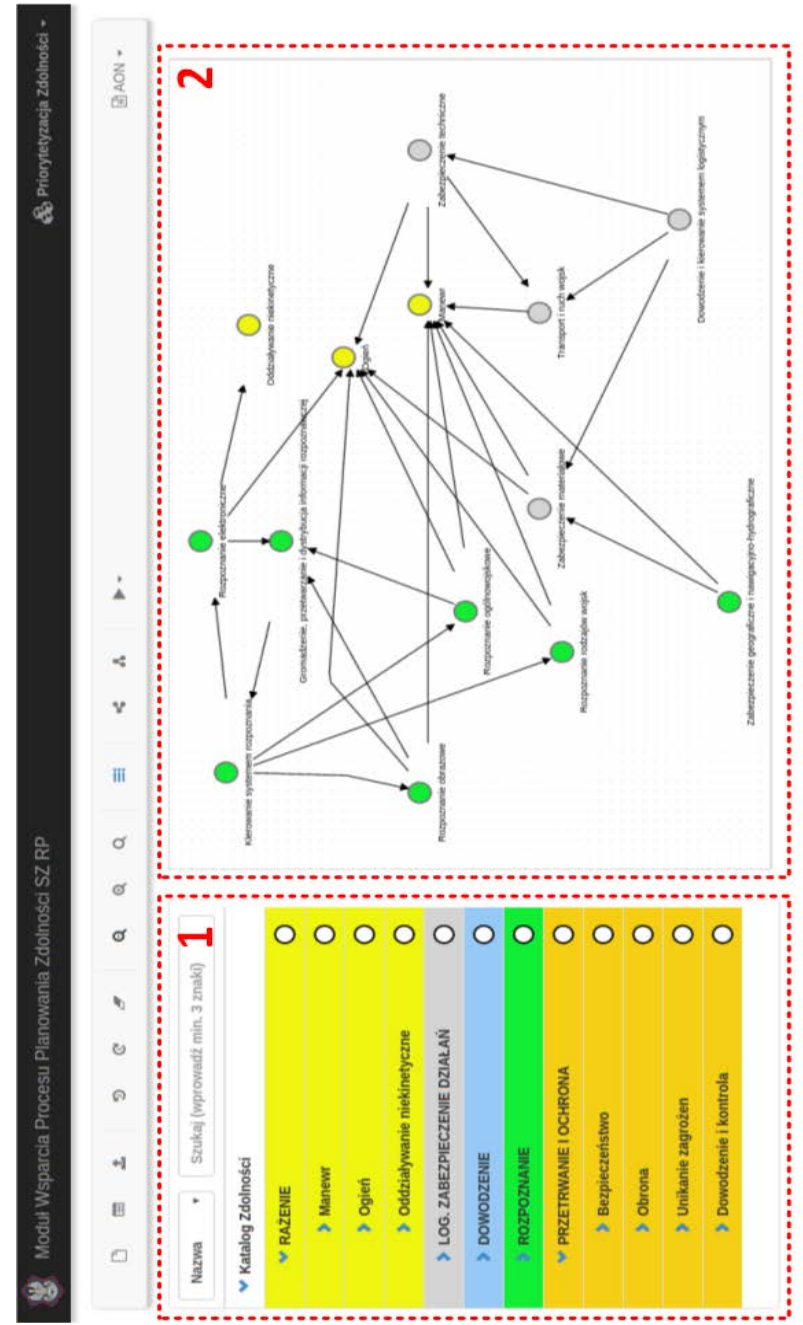

Fig. 3. Graphic interface of component - Prioritization of Capabilities

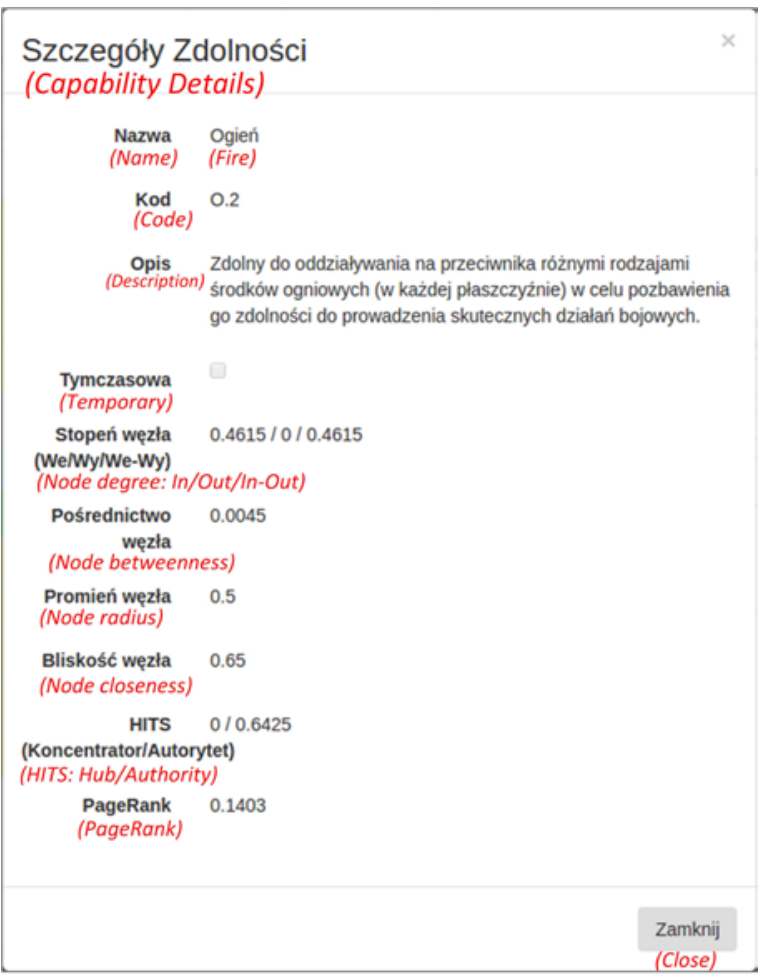

Fig. 4. Preview window of capability parameters 


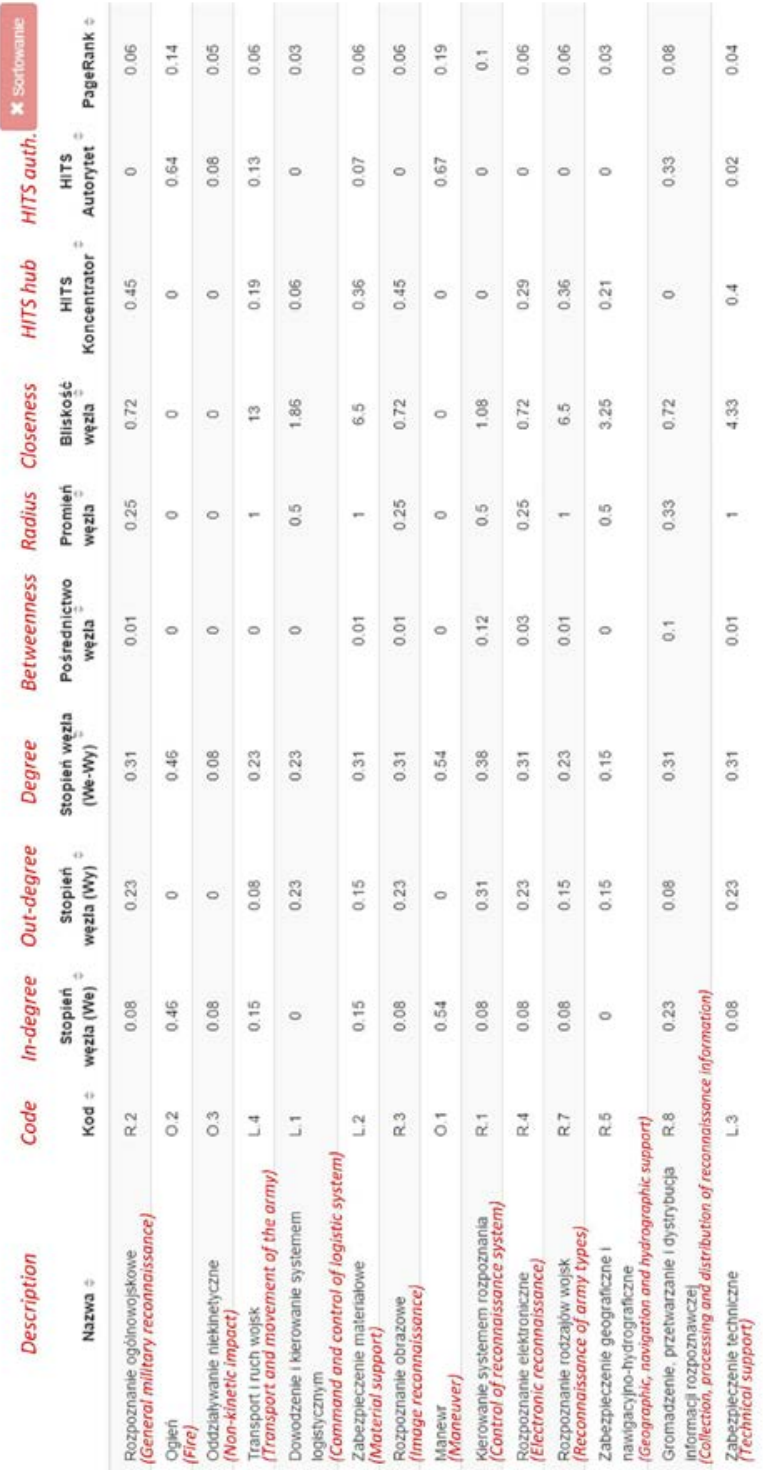

Fig. 5. Interactive table with the calculation results of the values of the centrality measures of capabilities

The structure of the capability dependency graph is quick to use and intuitive, as it operates on the basis of the drag and drop technique. It is required to choose one of the available capabilities and drag and drop it over the working area. It is possible to preview the parameters on each capability (Fig. 4), connect it with the other capability (buiding structure of capabilities interdependencies) or remove a choosen capability.

Once the design of the capability dependency graph is ready, it is possible to configure and initiate a number of algorithms for rating the vertices of the constructed graph. The algorithms are described in point 2.2. The calculation results are presented in the form of an interactive table (Fig. 5).

Furthermore, the values of particular centrality measures of capabilities may be exported to CSV file and saved for further analysis. Two methods for reapplying the design of the capability dependence graph are available in the Prioritization of Capabilities component. The methods are the following: entry in the database or export to the file, which may be later imported for the purpose of further analysis.

\section{Example of experiments}

To verify the developed method of prioritization of capabilities of the Armed Forces, a number of experiments were carried out. The capability dependency graphs developed in collaboration with the military experts, including employees of the War Studies University in Warsaw, constituted grounds for such experiments. Fig. 6 shows a model capability dependency graph for a specific Threat Reaction Scenario used in case of a High Intensity Conflict.

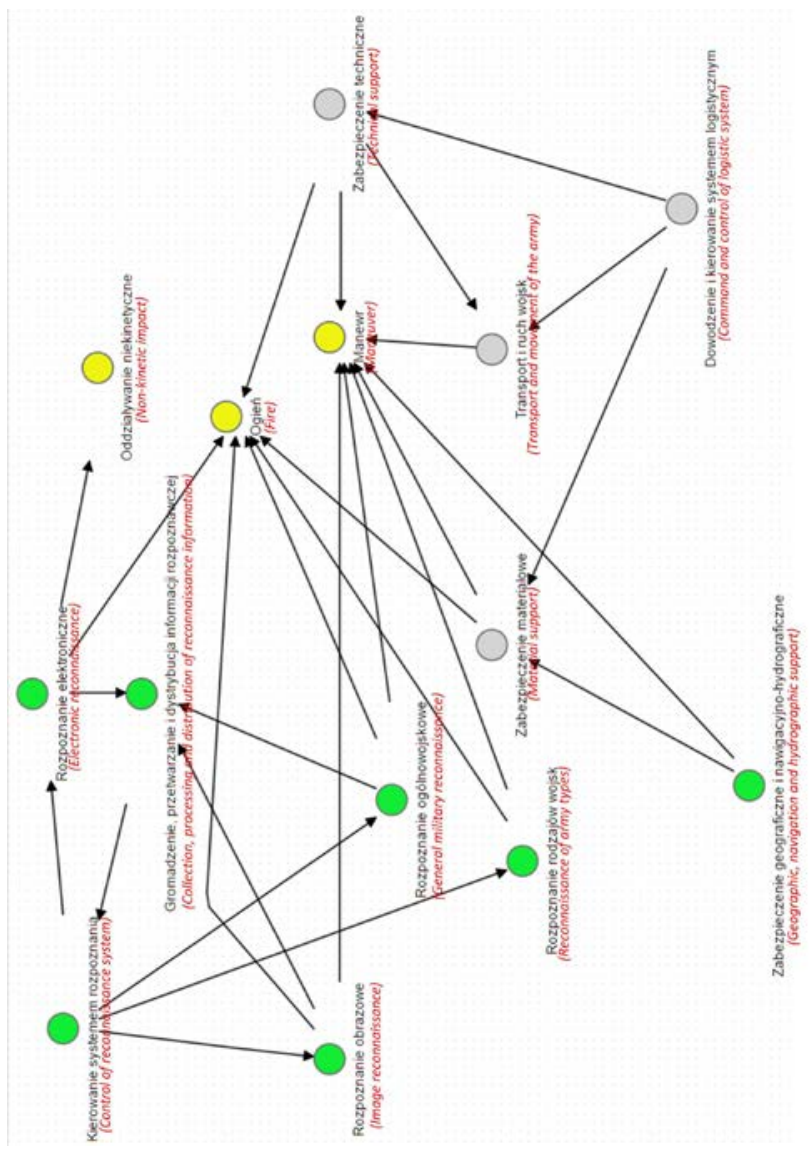

Fig. 6. Capability dependency graph for the High Intensity Conflict

The significance assessment of capabilities allows to obtain the following lists of the five most important capabilities (in descending order of importance), depending on the selected several centrality measures:

- in-degree of the vertice: Maneuver, Fire, Collection processing and distribution of reconnaissance information, Transport and movement of the troops, Material support;

- betweenness: Control of reconnaissance system, Collection processing and distribution of reconnaissance information, General military reconnaissance, Electronic reconnaissance, Image reconnaissance; 
- PageRank: Maneuver, Fire, Control of reconnaissance system, Collection, Processing and distribution of reconnaissance information, Material support;

- HITS - Authority centrality: Maneuver, Fire, Collection processing and distribution of reconnaissance information, Transport and movement of the troops, Non-kinetic impact;

- HITS - Hub centrality: General military reconnaissance, Image reconnaissance, Technical support, Material support, Reconnaissance of armed forces branches.

The obtained results, including their interpretation, were analyzed by military experts to validate the proposed method of prioritization of capabilities using the centrality measures. The conducted experiments confirmed the usefulness of such method, in particular by rationalizing the process of assigning priorities to capabilities thanks to the quantitative approach, which always objectivizes the rating.

\section{Summary}

The presented method of prioritization of capabilities of the Armed Forces constitutes an original proposal developed during the implementation of the project: "IT system supporting the capabilities development and identification of operational needs of the Armed Forces of the Republic of Poland" [3].

Special attention should be paid to the fact that the proposed method allows to perform a multidimensional significance assessment of capabilities. As a result, it is necessary to apply the method of multicriteria optimization in case of the decision-makers, who expect single-indicator functions of significance assessment of capabilities for the analysed Threat Reaction Scenario. If multiple Threat Reaction Scenarios are analysed, the capabilities required for the implementation of each such scenario must be prioritized independently, and then, the method of multicriteria optimization may be used for the global assessment of the capability priorities.

The proposed method may be naturally extended by replacing the capability dependency graph with the network of interdependencies between capabilities. This way it should be possible to reflect not only the structure, but also the level of interdependencies between capabilities, by introducing the function describing the dependency level on the CDG arcs. In such case, it is possible to use the modified method of similarity assessment of vertices in the network [7], [8].

Planning and programming process of the capabilities development of the Armed Forces of the Republic of Poland (see Fig. 1) contains two group of tasks: which are realized by human and only human (1) and which can be potentially automated (2). Therefore, we can use, in the further works, business process data flow approach between automated and human tasks [9].

\section{References}

1. Methodic of Planning and Programming Capabilities Development of Polish Armed Forces (Polish title: Metodyka Planowania i Programowania Rozwoju Zdolności SZ RP), Ministry of Defense, General Staff of Polish Armed Forces, Warsaw, Poland (2014)

2. P. Davis, Analytic Architecture for CapabilitiesBased Panning, Mission-System Analysis and Transformation, RAND, (2002)

3. A. Najgebauer, R. Antkiewicz, M. Chmielewski, M. Dyk, R. Kasprzyk, D. Pierzchała, J. Rulka, Z. Tarapata, The Qualitative and Quantitative Support Method for Capability Based Planning of Armed Forces Development, in: Intelligent Information and Database Systems, 7th Asian Conference, ACIIDS'2015, Springer International Publishing, Volume 9012, pp. 224-234, (2015)

4. U. Brandes, A Faster Algorithm for Betweenness Centrality, Journal of Mathematical Sociology, 25 (2), pp. 163-177, (2001)

5. R. Kasprzyk, Diffusion in Networks, Journal of Telecommunications and Information Technology, 2, pp. 99-106, (2012)

6. R. Kasprzyk, Complex Systems Evolution Models and Methods to Investigate Their Characteristics for Computer Identification of Potential Crises (Polish title: Modele ewolucji systemów złożonych i metody badania ich charakterystyk dla potrzeb komputerowej identyfikacji potencjalnych sytuacji kryzysowych), PhD thesis, Military University of Technology in Warsaw (2012)

7. Z. Tarapata, Multicriteria weighted graphs similarity and its application for decision situation pattern matching problem, Proceedings of the 13th IEEE/IFAC International Conference on Methods and Models in Automation and Robotics (MMAR'2007), pp.1149-1155, (2007)

8. Z. Tarapata, Models and algorithms for knowledgebased decision support and simulation in defense and transport applications, Habilitation thesis, Military University of Technology in Warsaw, Poland (2011)

9. M. Kiedrowicz, T. Nowicki, R. Waszkowski, Business Process Data Flow between Automated and Human Tasks, Proceedings of the 3rd International Conference on Social Science (ICSS 2016), vol. 1, ISBN: 978-1-60595-410-3, Shanghai, China (2016) 